International Electronic Journal of Algebra

VOLUME 21 (2017) 23-38

\title{
$H$-GALOIS EXTENSIONS WITH NORMAL BASIS FOR WEAK HOPF ALGEBRAS
}

\author{
J. N. Alonso Álvarez, J. M. Fernández Vilaboa and R. González Rodríguez \\ Received: 15 January 2016; Revised: 9 July 2016 \\ Communicated by A. Çiğdem Özcan
}

\begin{abstract}
Let $H$ be a weak Hopf algebra and let $A$ be an $H$-comodule algebra with subalgebra of coinvariants $A_{H}$. In this paper we introduce the notion of $H$-Galois extension with normal basis and we prove that $A_{H} \hookrightarrow A$ is an $H$-Galois extension with normal basis if and only if $A_{H} \hookrightarrow A$ is an $H$-cleft extension which admits a convolution invertible total integral. As a consequence, if $H$ is cocommutative and $A$ commutative, we obtain a bijective correspondence between the second cohomology group $H_{\varphi_{A_{H}}}^{2}\left(H, A_{H}\right)$ and the set of isomorphism classes of $H$-Galois extensions with normal basis whose left action over $A_{H}$ is $\varphi_{A_{H}}$.
\end{abstract}

Mathematics Subject Classification (2010): 18D10, 16T05

Keywords: $H$-Galois extensions, normal basis, weak Hopf algebra

\section{Introduction}

It is a well-known fact in classical Galois theory that if $B \subset A$ is a finite Galois extension of fields with Galois group $H$, then $A / B$ has a normal basis, i.e., there exists $a \in A$ such that the set $\{x . a ; x \in H\}$ is a basis for $A$ over $B$. Generalizing finite Galois extension of fields, Kreimer and Takeuchi introduce in [13] the notion of $H$-Galois extension with normal basis, associated to a Hopf algebra $H$ in a category of modules over a commutative ring, and in [10] Doi and Takeuchi show that there exists an equivalence between the notion of $H$-Galois extension with normal basis and the one of $H$-cleft extension for $H$. This result can be generalized to symmetric closed categories [11] and in [7] we find a more general formulation in the context of entwining structures that was extended to the weak setting in [2] by using the notion of weak $C$-cleft extensions defined in [1]. On the other hand, being $A$ an algebra, $C$ a coalgebra and $\Gamma_{H}^{A}: C \otimes A \rightarrow A \otimes C$ a morphism in a strict monoidal category with equalizers and coequalizers, such that $\left(A, C, \Gamma_{H}^{A}\right)$ is a weak entwining structure, we have introduced in [2] the notion of weak $C$-Galois extension with

This work was supported by Ministerio de Economía y Competitividad and by Feder founds. Grant MTM2013-43687-P: Homología, homotopía e invariantes categóricos en grupos y álgebras no asociativas. 
normal basis and we proved that, if $A \otimes-$ preserves coequalizers, there exists an equivalence between weak $C$-Galois extensions and weak $C$-cleft extensions. Taking into account that every right comodule algebra over a weak Hopf algebra $H$ induces a weak entwining structure, the results obtained in [1] and [2] can be applied for the study of Galois theory for weak Hopf algebras.

In [5] we introduce the notion of $H$-cleft extension for a weak Hopf algebra $H$ and we prove that this kind of extensions are examples of weak $H$-cleft extensions like the ones introduced in [1] and satisfying the classical notion of cleftness when particularizing to the Hopf setting. Assuming cocommutativity for $H$, we give in [5] a bijective correspondence between the equivalence classes of $H$-cleft extensions $A_{H} \hookrightarrow B$ and the equivalence classes of crossed systems for $H$ over $A_{H}$ where $A_{H}$ denotes the subalgebra of coinvariants of the $H$-comodule algebra $\left(A, \rho_{A}\right)$ in the weak context. This result permits to generalize the ones proved by Doi [9] about the characterization of equivalence classes of crossed systems as the second Sweedler cohomology group in the cocommutative Hopf algebra setting. To obtain this generalization we need the cohomology theory of algebras over cocommutative weak Hopf algebras we developed in [4] and used in [5] in order to give the weak Hopf version of Doi's result, i.e., a bijection between the isomorphism classes of $H$-cleft extensions $A_{H} \hookrightarrow B$, the equivalence classes of crossed systems for $H$ over $A_{H}$ and the second cohomology group $H_{\varphi_{\mathcal{Z}\left(A_{H}\right)}}^{2}\left(H, \mathcal{Z}\left(A_{H}\right)\right)$, where $\mathcal{Z}\left(A_{H}\right)$ is the center of $A_{H}$ and $\varphi_{\mathcal{Z}\left(A_{H}\right)}$ the corresponding associated action.

As we have pointed above, $H$-cleft extensions are a kind of weak $H$-cleft extensions, and these are equivalent to weak $H$-Galois extensions with normal basis. This leads naturally to the following question: Is there a special class of weak $H$-Galois extensions with normal basis equivalent to $H$-cleft extensions? In order to give an affirmative response to this question we introduce the notion of $H$-Galois extension with normal basis like a special kind of weak $H$-Galois extension with normal basis and we prove that if $A \otimes-$ preserves coequalizers, the following assertions are equivalent:

(i) $A_{H} \hookrightarrow A$ is an $H$-cleft extension that admits a convolution invertible total integral.

(ii) $A_{H} \hookrightarrow A$ is an $H$-Galois extension with normal basis.

As a consequence, taking into account that, if $H$ is cocommutative, every $H$ cleft extension $A_{H} \hookrightarrow A$ admits a convolution invertible total integral, we obtain that $A_{H} \hookrightarrow A$ is an $H$-cleft extension if and only if $A_{H} \hookrightarrow A$ is an $H$-Galois extension with normal basis. Therefore, if $A$ is commutative, we obtain a bijective correspondence between the second cohomology group $H_{\varphi_{A_{H}}}^{2}\left(H, A_{H}\right)$ and the set 
of equivalence classes of $H$-Galois extensions with normal basis with associated left action over the subalgebra of coinvariants $\varphi_{A_{H}}$.

\section{Galois extensions with normal basis and Cleft extensions in a weak setting}

Throughout this paper $\mathcal{C}=(\mathcal{C}, \otimes, K, c)$ is a symmetric monoidal category with equalizers and coequalizers, where $\otimes$ is the tensor product, $K$ the base object and $c_{M, N}: M \otimes N \rightarrow N \otimes M$ the natural isomorphism of symmetry. For any objects $A, B$ and $C$ in $\mathcal{C}$ the natural isomorphism $\mathfrak{a}_{A, B, C}:(A \otimes B) \otimes C \rightarrow A \otimes(B \otimes C)$ is called the associative constraint, and the natural isomorphisms $\mathfrak{l}_{A}: K \otimes A \rightarrow A$ and $\mathfrak{r}_{A}: A \otimes K \rightarrow A$, are known as the left and right unit constraints, respectively. Moreover, by Theorem XI.5.3 of [12] we know that every monoidal category is monoidally equivalent to a strict one (i.e., a category such that the constraint isomorphisms are identities), and then there is no loss of generality in assuming that $\mathcal{C}$ is strict.

We assume that the reader is familiar with the notions of (co)algebra and (co)module and morphisms between them in this monoidal setting (see [1], [2]). Note that if $\mathcal{C}$ admits equalizers then every idempotent morphism in $\mathcal{C}$ splits, i.e., for every morphism $q: Y \rightarrow Y$ such that $q=q \circ q$ there exists an object $Z$ (image of $q$ ) and morphisms $i: Z \rightarrow Y$ and $p: Y \rightarrow Z$ such that $q=i \circ p$ and $p \circ i=i d_{Z}$.

For each object $M$ in $\mathcal{C}$, we denote the identity morphism by $i d_{M}: M \rightarrow M$ and for simplicity of notation, given objects $M, N, P$ in $\mathcal{C}$ and a morphism $f: M \rightarrow N$, we write $P \otimes f$ for $i d_{P} \otimes f$ and $f \otimes P$ for $f \otimes i d_{P}$.

Let $D=\left(D, \varepsilon_{D}, \delta_{D}\right)$ be a coalgebra, with counit $\varepsilon_{D}: D \rightarrow K$ and coproduct $\delta_{D}: D \rightarrow D \otimes D$, and let $A=\left(A, \eta_{A}, \mu_{A}\right)$ be an algebra with unit $\eta_{A}: K \rightarrow A$ and product $\mu_{A}: A \otimes A \rightarrow A$. If $f, g: D \rightarrow A$ in $\mathcal{C}$ are morphisms in $\mathcal{C}, f * g$ denotes the usual convolution product in the category, that is, $f * g=\mu_{A} \circ(f \otimes g) \circ \delta_{D}$.

For an algebra $A$, the category of right (resp. left) $A$-modules will be denoted by $\mathcal{M}_{A}$ (resp. $\left.{ }_{A} \mathcal{M}\right)$. Similarly, if $D$ is a coalgebra we denote by $\mathcal{M}^{D}\left(\right.$ resp. $\left.{ }^{D} \mathcal{M}\right)$ the category of right (resp. left) $D$-comodules.

Definition 2.1. A weak bialgebra $H$ in $\mathcal{C}$ is an algebra $\left(H, \eta_{H}, \mu_{H}\right)$ and a coalgebra $\left(H, \varepsilon_{H}, \delta_{H}\right)$ satisfying:

$$
\begin{aligned}
\text { (a1) } & \delta_{H} \circ \mu_{H}=\left(\mu_{H} \otimes \mu_{H}\right) \circ\left(H \otimes c_{H, H} \otimes H\right) \circ\left(\delta_{H} \otimes \delta_{H}\right), \\
\text { (a2) } & \varepsilon_{H} \circ \mu_{H} \circ\left(\mu_{H} \otimes H\right)=\left(\varepsilon_{H} \otimes \varepsilon_{H}\right) \circ\left(\mu_{H} \otimes \mu_{H}\right) \circ\left(H \otimes \delta_{H} \otimes H\right) \\
& =\left(\varepsilon_{H} \otimes \varepsilon_{H}\right) \circ\left(\mu_{H} \otimes \mu_{H}\right) \circ\left(H \otimes\left(c_{H, H} \circ \delta_{H}\right) \otimes H\right), \\
\text { (a3) } & \left(\delta_{H} \otimes H\right) \circ \delta_{H} \circ \eta_{H}=\left(H \otimes \mu_{H} \otimes H\right) \circ\left(\delta_{H} \otimes \delta_{H}\right) \circ\left(\eta_{H} \otimes \eta_{H}\right) \\
& =\left(H \otimes\left(\mu_{H} \circ c_{H, H}\right) \otimes H\right) \circ\left(\delta_{H} \otimes \delta_{H}\right) \circ\left(\eta_{H} \otimes \eta_{H}\right) .
\end{aligned}
$$


If moreover,

(a4) there exists a morphism $\lambda_{H}: H \rightarrow H$ in $\mathcal{C}$ (called antipode of $H$ ) satisfying:

$(\mathrm{a} 4-1) i d_{H} * \lambda_{H}=\left(\left(\varepsilon_{H} \circ \mu_{H}\right) \otimes H\right) \circ\left(H \otimes c_{H, H}\right) \circ\left(\left(\delta_{H} \circ \eta_{H}\right) \otimes H\right)$,

$(\mathrm{a} 4-2) \lambda_{H} * i d_{H}=\left(H \otimes\left(\varepsilon_{H} \circ \mu_{H}\right)\right) \circ\left(c_{H, H} \otimes H\right) \circ\left(H \otimes\left(\delta_{H} \circ \eta_{H}\right)\right)$,

(a4-3) $\lambda_{H} * i d_{H} * \lambda_{H}=\lambda_{H}$.

we say that the weak bialgebra $H$ is a weak Hopf algebra in the category $\mathcal{C}$. Note that in a strict monoidal category the associativity of the convolution product follows by the associativity of the product $\mu_{H}$ and the coassociativity of the coproduct $\delta_{H}$.

In a similar way to the Hopf algebra case, the antipode $\lambda_{H}$ of a weak Hopf algebra $H$ is unique, antimultiplicative $\left(\lambda_{H} \circ \mu_{H}=\mu_{H} \circ\left(\lambda_{H} \otimes \lambda_{H}\right) \circ c_{H, H}\right)$, anticomultiplicative $\left(\delta_{H} \circ \lambda_{H}=c_{H, H} \circ\left(\lambda_{H} \otimes \lambda_{H}\right) \circ \delta_{H}\right)$ and leaves the unit $\eta_{H}$ and the counit $\varepsilon_{H}$ invariable $\left(\lambda_{H} \circ \eta_{H}=\eta_{H}, \varepsilon_{H} \circ \lambda_{H}=\varepsilon_{H}\right)$.

Moreover, we can define the idempotent morphisms $\Pi_{H}^{L}$ (target), $\Pi_{H}^{R}$ (source), $\bar{\Pi}_{H}^{L}$ and $\bar{\Pi}_{H}^{R}$ by

$$
\begin{aligned}
& \Pi_{H}^{L}=\left(\left(\varepsilon_{H} \circ \mu_{H}\right) \otimes H\right) \circ\left(H \otimes c_{H, H}\right) \circ\left(\left(\delta_{H} \circ \eta_{H}\right) \otimes H\right) ; \\
& \Pi_{H}^{R}=\left(H \otimes\left(\varepsilon_{H} \circ \mu_{H}\right)\right) \circ\left(c_{H, H} \otimes H\right) \circ\left(H \otimes\left(\delta_{H} \circ \eta_{H}\right)\right) ; \\
& \bar{\Pi}_{H}^{L}=\left(H \otimes\left(\varepsilon_{H} \circ \mu_{H}\right)\right) \circ\left(\left(\delta_{H} \circ \eta_{H}\right) \otimes H\right) ; \\
& \bar{\Pi}_{H}^{R}=\left(\left(\varepsilon_{H} \circ \mu_{H}\right) \otimes H\right) \circ\left(H \otimes\left(\delta_{H} \circ \eta_{H}\right)\right) ;
\end{aligned}
$$

which satisfy the equalities $\Pi_{H}^{L}=i d_{H} * \lambda_{H}, \Pi_{H}^{R}=\lambda_{H} * i d_{H}$ and then $\Pi_{H}^{L} * \Pi_{H}^{L}=\Pi_{H}^{L}$, $\Pi_{H}^{R} * \Pi_{H}^{R}=\Pi_{H}^{R}$. In what follows we denote by $H_{L}$ the image of the target morphism and by $p_{L}$ and $i_{L}$ the morphisms such that $i_{L} \circ p_{L}=\Pi_{H}^{L}$ and $p_{L} \circ i_{L}=i d_{H_{L}}$.

Finally, we have that (see [6]),

$$
\begin{aligned}
& \Pi_{H}^{L} \circ \bar{\Pi}_{H}^{L}=\Pi_{H}^{L} ; \quad \Pi_{H}^{L} \circ \bar{\Pi}_{H}^{R}=\bar{\Pi}_{H}^{R} ; \quad \Pi_{H}^{R} \circ \bar{\Pi}_{H}^{L}=\bar{\Pi}_{H}^{L} ; \quad \Pi_{H}^{R} \circ \bar{\Pi}_{H}^{R}=\Pi_{H}^{R} ; \\
& \bar{\Pi}_{H}^{L} \circ \Pi_{H}^{L}=\bar{\Pi}_{H}^{L} ; \quad \bar{\Pi}_{H}^{L} \circ \Pi_{H}^{R}=\Pi_{H}^{R} ; \quad \bar{\Pi}_{H}^{R} \circ \Pi_{H}^{L}=\Pi_{H}^{L} ; \quad \bar{\Pi}_{H}^{R} \circ \Pi_{H}^{R}=\bar{\Pi}_{H}^{R} .
\end{aligned}
$$

Definition 2.2. Let $H$ be a weak bialgebra and let $A$ be an algebra with coaction $\rho_{A}: A \rightarrow A \otimes H$ such that $\left(A, \rho_{A}\right)$ is a right $H$-comodule satisfying the equality $\mu_{A \otimes H} \circ\left(\rho_{A} \otimes \rho_{A}\right)=\rho_{A} \circ \mu_{A}$. The object $\left(A, \rho_{A}\right)$ is called a right $H$-comodule algebra if one of the following equivalent conditions holds (see [8], Proposition 4.10):

(b1) $\left(\rho_{A} \otimes H\right) \circ \rho_{A} \circ \eta_{A}=\left(A \otimes\left(\mu_{H} \circ c_{H, H}\right) \otimes H\right) \circ\left(\left(\rho_{A} \circ \eta_{A}\right) \otimes\left(\delta_{H} \circ \eta_{H}\right)\right)$,

(b2) $\left(\rho_{A} \otimes H\right) \circ \rho_{A} \circ \eta_{A}=\left(A \otimes \mu_{H} \otimes H\right) \circ\left(\left(\rho_{A} \circ \eta_{A}\right) \otimes\left(\delta_{H} \circ \eta_{H}\right)\right)$,

(b3) $\left(A \otimes \bar{\Pi}_{H}^{R}\right) \circ \rho_{A}=\left(\mu_{A} \otimes H\right) \circ\left(A \otimes\left(\rho_{A} \circ \eta_{A}\right)\right)$,

(b4) $\left(A \otimes \Pi_{H}^{L}\right) \circ \rho_{A}=\left(\mu_{A} \otimes H\right) \circ\left(A \otimes c_{H, A}\right) \circ\left(\left(\rho_{A} \circ \eta_{A}\right) \otimes A\right)$,

(b5) $\left(A \otimes \bar{\Pi}_{H}^{R}\right) \circ \rho_{A} \circ \eta_{A}=\rho_{A} \circ \eta_{A}$,

(b6) $\left(A \otimes \Pi_{H}^{L}\right) \circ \rho_{A} \circ \eta_{A}=\rho_{A} \circ \eta_{A}$. 
For a right $H$-comodule algebra $A$ we define $\Gamma_{A}^{H}: H \otimes A \rightarrow A \otimes H$ as $\Gamma_{A}^{H}=$ $\left(A \otimes \mu_{H}\right) \circ\left(c_{H, A} \otimes H\right) \circ\left(H \otimes \rho_{A}\right)$. Then the triple $\left(A, H, \Gamma_{A}^{H}\right)$ is a right-right weak entwining structure (see [8], Theorem 4.14), i.e., it satisfies

$$
\begin{gathered}
\Gamma_{A}^{H} \circ\left(H \otimes \mu_{A}\right)=\left(\mu_{A} \otimes H\right) \circ\left(A \otimes \Gamma_{A}^{H}\right) \circ\left(\Gamma_{A}^{H} \otimes A\right), \\
\left(A \otimes \delta_{H}\right) \circ \Gamma_{A}^{H}=\left(\Gamma_{A}^{H} \otimes H\right) \circ\left(H \otimes \Gamma_{A}^{H}\right) \circ\left(\delta_{H} \otimes A\right), \\
\Gamma_{A}^{H} \circ\left(H \otimes \eta_{A}\right)=\left(e_{A} \otimes H\right) \circ \delta_{H}, \\
\left(A \otimes \varepsilon_{H}\right) \circ \Gamma_{A}^{H}=\mu_{A} \circ\left(e_{A} \otimes A\right),
\end{gathered}
$$

where

$$
e_{A}=\left(A \otimes \varepsilon_{H}\right) \circ \Gamma_{A}^{H} \circ\left(H \otimes \eta_{A}\right) .
$$

Let $A$ and $H$ be fixed. We denote by $\mathcal{M}_{A}^{H}\left(\Gamma_{A}^{H}\right)$ the category of right-right weak entwined modules, i.e., the objects $M$ in $\mathcal{C}$ together with two morphisms $\phi_{M}: M \otimes A \rightarrow A$ and $\rho_{M}: M \rightarrow M \otimes H$ such that $\left(M, \phi_{M}\right)$ is a right $A$-module, $\left(M, \rho_{M}\right)$ is a right $H$-comodule and the following equality

$$
\rho_{M} \circ \phi_{M}=\left(\phi_{M} \otimes H\right) \circ\left(M \otimes \Gamma_{A}^{H}\right) \circ\left(\rho_{M} \otimes A\right)
$$

holds. Obviously, if $\left(A, \rho_{A}\right)$ is a right $H$-comodule algebra, $\left(A, \mu_{A}, \rho_{A}\right)$ is an object of $\mathcal{M}_{A}^{H}\left(\Gamma_{A}^{H}\right)$.

Let $\left(A, \rho_{A}\right)$ be a right $H$-comodule algebra. We define the subalgebra of coinvariants of $A$ by the equalizer:

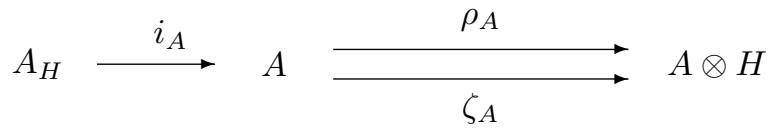

where $\zeta_{A}=\left(\mu_{A} \otimes H\right) \circ\left(A \otimes\left(\rho_{A} \circ \eta_{A}\right)\right)$. Note that, by (b3), $\zeta_{A}=\left(A \otimes \bar{\Pi}_{H}^{R}\right) \circ \rho_{A}$ and also, by (1) and (2), $\left(A_{H}, i_{A}\right)$ is the equalizer of $\rho_{A}$ and $\left(A \otimes \Pi_{H}^{L}\right) \circ \rho_{A}$.

It is not difficult to see that $\left(A_{H}, \eta_{A_{H}}, \mu_{A_{H}}\right)$ is an algebra, being $\eta_{A_{H}}$ and $\mu_{A_{H}}$ the factorizations through the equalizer $i_{A}$ of the morphisms $\eta_{A}$ and $\mu_{A} \circ\left(i_{A} \otimes i_{A}\right)$, respectively. As a consequence, $\varphi_{A}=\mu_{A} \circ\left(i_{A} \otimes A\right)\left(\right.$ respectively $\left.\phi_{A}=\mu_{A} \circ\left(A \otimes i_{A}\right)\right)$ defines a left (right) $A_{H}$-module structure for $A$.

Note that the weak Hopf algebra $H$ is a right $H$-comodule algebra with comodule structure giving by $\rho_{H}=\delta_{H}$ and subalgebra of coinvariants $H_{H}=H_{L}$. In this case $i_{H}=i_{L}$.

The morphism $\Delta_{A \otimes H}=\left(\mu_{A} \otimes H\right) \circ\left(A \otimes \Gamma_{A}^{H}\right) \circ\left(A \otimes H \otimes \eta_{A}\right): A \otimes H \rightarrow A \otimes H$ is an idempotent and, as a consequence, there exist an object $A \square H$ and morphisms $i_{A \otimes H}: A \square H \rightarrow A \otimes H, p_{A \otimes H}: A \otimes H \rightarrow A \square H$ such that $\Delta_{A \otimes H}=i_{A \otimes H} \circ$ $p_{A \otimes H}$ and $i d_{A \square H}=p_{A \otimes H} \circ i_{A \otimes H}$. Moreover $A \square H$ is a right $A$-module, where the action is defined by $\phi_{A \square H}=p_{A \otimes H} \circ\left(\mu_{A} \otimes H\right) \circ\left(A \otimes \Gamma_{A}^{H}\right) \circ\left(i_{A \otimes H} \otimes A\right)$, and a 
right $H$-comodule, with coaction $\rho_{A \square H}=\left(p_{A \otimes H} \otimes H\right) \circ\left(A \otimes \delta_{H}\right) \circ i_{A \otimes H}$, and $\left(A \square H, \phi_{A \square H}, \rho_{A \square H}\right)$ is a weak entwined module and a left $A$-module with action $\varphi_{A \square H}=p_{A \otimes H} \circ\left(\mu_{A} \otimes H\right) \circ\left(A \otimes i_{A \otimes H}\right)$. On the other hand, the equality

$$
\Delta_{A \otimes H}=\left(\mu_{A} \otimes H\right) \circ\left(A \otimes\left(\left(e_{A} \otimes H\right) \circ \delta_{H}\right)\right),
$$

comes directly from (5).

The morphism (lifted canonical morphism) $r_{A}=p_{A \otimes H} \circ\left(\mu_{A} \otimes H\right) \circ\left(A \otimes \rho_{A}\right)$ : $A \otimes A \rightarrow A \square H$ factorizes through the coequalizer morphism $q_{A, A}: A \otimes A \rightarrow A \otimes_{A_{H}} A$ of the morphisms $\theta_{A, A}^{1}=A \otimes \varphi_{A}$ and $\theta_{A, A}^{2}=\phi_{A} \otimes A$. As a consequence, there exists a unique morphism, called the canonical morphism, $\gamma_{A}: A \otimes_{A_{H}} A \rightarrow A \square H$ such that $\gamma_{A} \circ q_{A, A}=r_{A}$. Further, $r_{A}$ and $\gamma_{A}$ are morphisms of right $H$-comodules being $\rho_{A \otimes A}=A \otimes \rho_{A}$ and $\rho_{A \otimes_{A_{H}} A}$ the factorization of $\left(q_{A, A} \otimes H\right) \circ\left(A \otimes \rho_{A}\right)$ through the coequalizer $q_{A, A}$. If the functor $A \otimes-$ preserves coequalizers, $\gamma_{A}$ is a morphism of left $A$-modules where $\varphi_{A \otimes_{B} A}$ is the factorization of $q_{A, A} \circ\left(\mu_{A} \otimes A\right)$ through the coequalizer $A \otimes q_{A, A}$. Finally, $\gamma_{A}$ is a morphism of right $A$-modules where $\phi_{A \otimes_{B} A}$ is the factorization of $q_{A, A} \circ\left(A \otimes \mu_{A}\right)$ through the coequalizer $q_{A, A} \otimes A$.

Definition 2.3. If the functor $A \otimes-$ preserves coequalizers, we say that $A_{H} \hookrightarrow A$ is a weak $H$-Galois extension if the canonical morphism $\gamma_{A}$ is an isomorphism.

Note that, if $\mathcal{C}$ is a closed category, the functor $A \otimes-$ preserves coequalizers. Also, if $A$ is a finite object, i.e., there exists an object $A^{*}$ and an adjunction $A \otimes-\dashv$ $A^{*} \otimes-$, we have that $A \otimes-$ preserves coequalizers.

Let $H$ be a weak Hopf algebra and let $\left(A, \rho_{A}\right)$ be a right $H$-comodule algebra. In Definition 1.8 of [1] we introduce the set $\operatorname{Reg}^{W R}(H, A)$ as the one whose elements are the morphisms $h: H \rightarrow A$ such that there exists $h^{-1}: H \rightarrow A$, called the left weak inverse of $h$, satisfying $h^{-1} * h=e_{A}$ where $e_{A}$ is the morphism defined in (7) for the right-right weak entwining structure $\Gamma_{A}^{H}$ associated to $\left(A, \rho_{A}\right)$.

Definition 2.4. We say that $A_{H} \hookrightarrow A$ is a weak $H$-cleft extension if there exists a morphism $h: H \rightarrow A$ in $\operatorname{Reg}^{W R}(H, A)$ (called the cleaving morphism) of right $H$-comodules such that

$$
\Gamma_{A}^{H} \circ\left(H \otimes h^{-1}\right) \circ \delta_{H}=\zeta_{A} \circ\left(e_{A} * h^{-1}\right) .
$$

Also, by (2.9) of [5], we can assume without loss of generality that $e_{A} * h^{-1}=h^{-1}$ and as a consequence (10) can be expressed as

$$
\Gamma_{A}^{H} \circ\left(H \otimes h^{-1}\right) \circ \delta_{H}=\zeta_{A} \circ h^{-1} .
$$

Then, if the extension $A_{H} \hookrightarrow A$ is weak $H$-cleft, by Proposition 1.12 of [1], we get that $q_{A}=\mu_{A} \circ\left(A \otimes h^{-1}\right) \circ \rho_{A}: A \rightarrow A$ factorizes through $i_{A}$. Therefore, 
there exists a unique morphism $p_{A}: A \rightarrow A_{H}$ such that $q_{A}=i_{A} \circ p_{A}$. Then, $h * h^{-1}=q_{A} \circ h$ and, as a consequence, $h * h^{-1}$ admits a factorization through $i_{A}$.

Now we recall the definition of $H$-Galois extension with normal basis we formulate in [2].

Definition 2.5. A weak $H$-Galois extension $A_{H} \hookrightarrow A$ has a normal basis if there exists an idempotent morphism of left $A_{H}$-modules and right $H$-comodules $\Omega_{A_{H} \otimes H}$ : $A_{H} \otimes H \rightarrow A_{H} \otimes H\left(\varphi_{A_{H} \otimes H}=\mu_{A_{H}} \otimes H, \rho_{A_{H} \otimes H}=A_{H} \otimes \delta_{H}\right)$ and an isomorphism of left $A_{H}$-modules and right $H$-comodules $b_{A}: A \rightarrow A_{H} \times H$, where $A_{H} \times H$ is the image of $\Omega_{A_{H} \otimes H}$ and $\varphi_{A_{H} \times H}=p_{A_{H}, H} \circ \varphi_{A_{H} \otimes H} \circ\left(A_{H} \otimes i_{A_{H}, H}\right), \rho_{A_{H} \times H}=\left(p_{A_{H}, H} \otimes\right.$ $H) \circ \rho_{A_{H} \otimes H} \circ i_{A_{H}, H}$, being $i_{A_{H}, H}: A_{H} \times H \rightarrow A_{H} \otimes H$ and $p_{A_{H}, H}: A_{H} \otimes H \rightarrow A_{H} \times$ $H$ the morphisms such that $i_{A_{H}, H} \circ p_{A_{H}, H}=\Omega_{A_{H} \otimes H}$ and $p_{A_{H}, H} \circ i_{A_{H}, H}=i d_{A_{H} \times H}$.

For a weak $H$-Galois extension with normal basis, if we define $\omega_{A}=b_{A}^{-1} \circ p_{A_{H}, H}$ : $A_{H} \otimes H \rightarrow A$ and $\omega_{A}^{\prime}=i_{A_{H}, H} \circ b_{A}: A \rightarrow A_{H} \otimes H$, the morphism $\omega_{A}^{\prime} \circ \omega_{A}=\Omega_{A_{H} \otimes H}$, $\omega_{A} \circ \omega_{A}^{\prime}=i d_{A}$ and $m_{A}^{\prime}=\mu_{A} \circ\left(A \otimes\left(\left(i_{A} \otimes \varepsilon_{H}\right) \circ \omega_{A}^{\prime}\right)\right): A \otimes A \rightarrow A$ factorizes through the coequalizer $q_{A, A}$. Then there exists a unique morphism of left $A$ modules $m_{A}: A \otimes_{A_{H}} A \rightarrow A$ such that

$$
m_{A} \circ q_{A, A}=\mu_{A} \circ\left(A \otimes\left(\left(i_{A} \otimes \varepsilon_{H}\right) \circ \omega_{A}^{\prime}\right)\right)
$$

(see Lemma 1.9 of [2]). Note that, in these conditions, we have that $p_{A_{H}, H}, i_{A_{H}, H}$, $\omega_{A}$ and $\omega_{A}^{\prime}$ are also morphisms of left $A_{H}$-modules and right $H$-comodules.

As we have showed in [1], there is a close connection between weak $H$-cleft extensions and weak $H$-Galois extensions with normal basis. More precisely, the main result in [1] establishes that, if $A \otimes-$ preserves coequalizers, $A_{H} \hookrightarrow A$ is a weak $H$-cleft extension if and only if $A_{H} \hookrightarrow A$ is a weak $H$-Galois extension with normal basis. For clarity we briefly review the proof:

Let $A_{H} \hookrightarrow A$ be a weak $H$-Galois extension with normal basis. We define the cleaving morphism $h_{A}=\omega_{A} \circ\left(\eta_{A_{H}} \otimes H\right): H \rightarrow A$ and its left weak inverse is $h_{A}^{-1}=m_{A} \circ \gamma_{A}^{-1} \circ p_{A \otimes H} \circ\left(\eta_{A} \otimes H\right): H \rightarrow A$. Note that in this part of the proof we obtain that $m_{A} \circ \gamma_{A}^{-1} \circ p_{A \otimes C} \circ \rho_{A}=\left(\left(i_{C}^{A} \otimes \varepsilon_{C}\right) \circ \omega_{A}^{\prime}\right.$ and

$$
h_{A} * h_{A}^{-1}=\left(i_{A} \otimes \varepsilon_{H}\right) \circ \Omega_{A_{H} \otimes H} \circ\left(\eta_{A_{H}} \otimes H\right) .
$$

Conversely, if $A_{H} \hookrightarrow A$ is a weak $H$-cleft extension with cleaving morphism $h$, the morphisms of left $A_{H^{-}}$-modules and right $H$-comodules defined by $\omega_{A}=\mu_{A} \circ\left(i_{A} \otimes h\right)$ and $\omega_{A}^{\prime}=\left(p_{A} \otimes H\right) \circ \rho_{A}$ satisfy the equality $\omega_{A} \circ \omega_{A}^{\prime}=i d_{A}$. As a consequence, the morphism $\Omega_{A_{H} \otimes H}=\omega_{A}^{\prime} \circ \omega_{A}$ is idempotent and we have a commutative diagram 


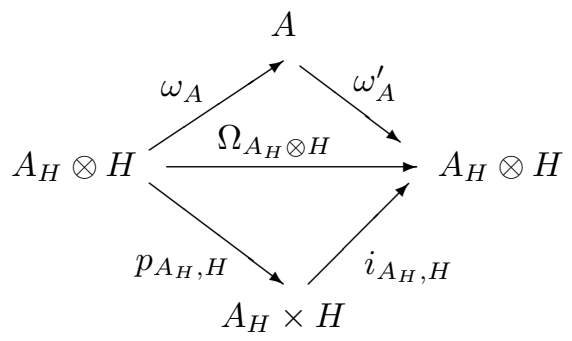

where $p_{A_{H}, H} \circ i_{A_{H}, H}=i d_{A_{H} \times H}$. Therefore, the morphism $b_{A}=p_{A_{H}, H} \circ \omega_{A}^{\prime}$ is an isomorphism of right $H$-comodules and left $A_{H}$-modules with inverse $b_{A}^{-1}=$ $\omega_{A} \circ i_{A_{H}, H}$. Moreover, the inverse of the canonical morphism $\gamma_{A}$ is $\gamma_{A}^{-1}=q_{A, A}$ 。 $\left(\mu_{A} \otimes A\right) \circ\left(A \otimes h_{A}^{-1} \otimes h_{A}\right) \circ\left(A \otimes \delta_{H}\right) \circ i_{A \otimes H}$.

In the second section of [5], we introduce the notion of $H$-cleft extension for a weak Hopf algebra $H$ and we prove that this kind of extensions are examples of weak $H$-cleft extensions. To define $H$-cleft extensions we need convolution invertible integrals. As in the Hopf setting, for a weak Hopf algebra $H$ and a right $H$-comodule algebra $\left(A, \rho_{A}\right)$, an integral is a morphism of right $H$-comodules $f: H \rightarrow A$. If moreover $f \circ \eta_{H}=\eta_{A}$ we will say that the integral is total.

An integral $f: H \rightarrow A$ is convolution invertible if there exists a morphism $f^{-1}: H \rightarrow A$ (called the convolution inverse of $f$ ) such that

(c1) $f^{-1} * f=e_{A}$.

(c2) $f * f^{-1}=\left(A \otimes\left(\varepsilon_{H} \circ \mu_{H}\right)\right) \circ\left(\left(\rho_{A} \circ \eta_{A}\right) \otimes H\right)$.

(c3) $f^{-1} * f * f^{-1}=f^{-1}$.

Trivially, the inverse is unique and we get that $f * f^{-1} * f=f$ (see Definition 2.4 of [5]). Note that, when $f$ is a total integral, we can rewrite (c1) as $f^{-1} * f=f \circ \Pi_{H}^{R}$ and $(\mathrm{c} 2)$ as $f * f^{-1}=f \circ \bar{\Pi}_{H}^{L}$.

Definition 2.6. We say that $A_{H} \hookrightarrow A$ is an $H$-cleft extension if there exists a convolution invertible integral $h: H \rightarrow A$ such that the morphism $h * h^{-1}$ factorizes through the equalizer $i_{A}$.

Obviously, $H_{L} \hookrightarrow H$ is a weak $H$-cleft extension with $h=i d_{H}$ and $h^{-1}=\lambda_{H}$. By Proposition 2.2 of [5] we know that if $H$ is a cocommutative weak Hopf algebra and there exists a convolution invertible integral $f: H \rightarrow A$ then $A_{H} \hookrightarrow A$ is an $H$-cleft extension. Also, by Corollary 2.1 of [5], we have that an $H$-cleft extension is also a weak $H$-cleft extension. Finally, Proposition 2.3 of [5] asserts that, if the antipode of $H$ is an isomorphism and $A_{H} \hookrightarrow A$ is an $H$-cleft extension with convolution invertible integral $f$, then $\left.h=\mu_{A} \circ\left(f \otimes\left(f^{-1} \circ \eta_{H}\right)\right)\right)$ is a total integral. Moreover, if $H$ is cocommutative $h$ is convolution invertible. 
In the following definition we introduce the notion of $H$-Galois extension with normal basis.

Definition 2.7. Let $H$ be a weak Hopf algebra and let $\left(A, \rho_{A}\right)$ be a right $H$-comodule algebra such that $A \otimes-$ preserves coequalizers. We say that a weak $H$-Galois extension with normal basis $A_{H} \hookrightarrow A$ is an $H$-Galois extension with normal basis if the following identities hold:

(d1) $b_{A} \circ \eta_{A}=p_{A_{H}, H} \circ\left(\eta_{A_{H}} \otimes \eta_{H}\right)$.

$(\mathrm{d} 2)\left(\left(b_{A} \circ i_{A}\right) \otimes \varepsilon_{H}\right) \circ \Omega_{A_{H} \otimes H} \circ\left(\eta_{A_{H}} \otimes H\right)=p_{A_{H}, H} \circ\left(\eta_{A_{H}} \otimes \bar{\Pi}_{H}^{L}\right)$.

\section{Galois and Cleft extensions and cohomology}

In this section we give the main results of the paper and a cohomological interpretation of Cleft extensions.

Lemma 3.1. Let $H$ be a weak Hopf algebra and let $\left(A, \rho_{A}\right)$ be a right $H$-comodule algebra such that $A \otimes-$ preserves coequalizers. If $A_{H} \hookrightarrow A$ is an $H$-Galois extension with normal basis the following equality holds:

$$
\left(A_{H} \otimes \varepsilon_{H}\right) \circ \Omega_{A_{H} \otimes H} \circ\left(A_{H} \otimes \eta_{H}\right)=i d_{A_{H}} .
$$

Proof. First of all, note that by the definition of the morphism $\bar{\Pi}_{H}^{L}$ and the properties of the (co)unit $\eta_{H}\left(\varepsilon_{H}\right)$, it is easy to see that $\bar{\Pi}_{H}^{L} \circ \eta_{H}=\eta_{H}$. Now, by composing (d2) of Definition 2.7 with $\eta_{H}$ and using (d1) we have that

$$
\left(\left(b_{A} \circ i_{A}\right) \otimes \varepsilon_{H}\right) \circ \Omega_{A_{H} \otimes H} \circ\left(\eta_{A_{H}} \otimes \eta_{H}\right)=p_{A_{H}, H} \circ\left(\eta_{A_{H}} \otimes \eta_{H}\right)=b_{A} \circ \eta_{A} .
$$

Therefore,

$$
\left(i_{A} \otimes \varepsilon_{H}\right) \circ \Omega_{A_{H} \otimes H} \circ\left(\eta_{A_{H}} \otimes \eta_{H}\right)=\eta_{A}
$$

holds and as a consequence we have:

$$
\begin{aligned}
& i_{A} \\
= & \mu_{A} \circ\left(i_{A} \otimes \eta_{A}\right) \\
= & \left(\left(\mu_{A} \circ\left(i_{A} \otimes i_{A}\right) \otimes \varepsilon_{H}\right) \circ\left(A_{H} \otimes\left(\Omega_{A_{H} \otimes H} \circ\left(\eta_{A_{H}} \otimes \eta_{H}\right)\right)\right)\right. \\
= & \left(i_{A} \otimes \varepsilon_{H}\right) \circ\left(\mu_{A_{H}} \otimes H\right) \circ\left(A_{H} \otimes\left(\Omega_{A_{H} \otimes H} \circ\left(\eta_{A_{H}} \otimes \eta_{H}\right)\right)\right) \\
= & \left(i_{A} \otimes \varepsilon_{H}\right) \circ \Omega_{A_{H} \otimes H} \circ\left(A_{H} \otimes \eta_{H}\right) .
\end{aligned}
$$

where the first equality follows by the properties of the unit $\eta_{A}$, the second one by (15), the third one by the properties of $\mu_{A_{H}}$, the fourth one because $\Omega_{A_{H} \otimes H}$ is a morphism of left $A_{H}$-modules.

Then, using that $i_{A}$ is a monomorphism we conclude the proof.

Theorem 3.2. Let $H$ be a weak Hopf algebra and let $\left(A, \rho_{A}\right)$ be a right $H$-comodule algebra such that $A \otimes-$ preserves coequalizers. The following are equivalent. 
(i) $A_{H} \hookrightarrow A$ is an $H$-cleft extension that admits a convolution invertible total integral.

(ii) $A_{H} \hookrightarrow A$ is an $H$-Galois extension with normal basis.

Proof. (i) $\Rightarrow$ (ii) By Corollary 2.1 of [5] we know that if $A_{H} \hookrightarrow A$ is an $H$-cleft extension with convolution invertible integral $h$ then it is a weak $H$-cleft extension with cleaving morphism $h$. Therefore, by Theorem 2.11 of [2], we obtain that $A_{H} \hookrightarrow A$ is a weak $H$-Galois extension with normal basis where $\omega_{A}=\mu_{A} \circ\left(i_{A} \otimes h\right)$, $\omega_{A}^{\prime}=\left(p_{A} \otimes H\right) \circ \rho_{A}, \Omega_{A_{H} \otimes H}=\omega_{A}^{\prime} \circ \omega_{A}, b_{A}=p_{A_{H}, H} \circ \omega_{A}^{\prime}$ and $b_{A}^{-1}=\omega_{A} \circ i_{A_{H}, H}$. Moreover, for $\Omega_{A_{H} \otimes H}$ we have the following identity:

$$
\Omega_{A_{H} \otimes H}=\left(\left(p_{A} \circ \mu_{A} \circ\left(i_{A} \otimes h\right)\right) \otimes H\right) \circ\left(A_{H} \otimes \delta_{H}\right) .
$$

Indeed:

$$
\begin{aligned}
& \Omega_{A_{H} \otimes H} \\
= & \left(p_{A} \otimes H\right) \circ \mu_{A \otimes H} \circ\left(\left(\rho_{A} \circ i_{A}\right) \otimes\left(\rho_{A} \circ h\right)\right) \\
= & \left(p_{A} \otimes H\right) \circ \mu_{A \otimes H} \circ\left(\left(\zeta_{A} \circ i_{A}\right) \otimes\left(\rho_{A} \circ h\right)\right) \\
= & \left(\left(p_{A} \circ \mu_{A}\right) \otimes H\right) \circ\left(i_{A} \otimes\left(\mu_{A \otimes H} \circ\left(\left(\rho_{A} \circ \eta_{A}\right) \otimes\left(\rho_{A} \circ h\right)\right)\right)\right) \\
= & \left(\left(p_{A} \circ \mu_{A}\right) \otimes H\right) \circ\left(i_{A} \otimes\left(\rho_{A} \circ h\right)\right) \\
= & \left(\left(p_{A} \circ \mu_{A}\right) \otimes H\right) \circ\left(i_{A} \otimes\left((h \otimes H) \circ \delta_{H}\right)\right) \\
= & \left(\left(\mu_{A_{H}} \circ\left(A_{H} \otimes\left(p_{A} \circ h\right)\right)\right) \otimes H\right) \circ\left(A_{H} \otimes \delta_{H}\right),
\end{aligned}
$$

where the first equality follows by the structure of right $H$-comodule algebra of $A$, the second one by the definition of $A_{H}$, the third one by the associativity of $\mu_{A}$, the fourth one by the structure of right $H$-comodule algebra of $A$ and the properties of the unity $\eta_{A}$. In the fifth equality we used that $h$ is a right $H$-comodule morphism and finally, the last one follows by (e1) of Lemma 3.11 of [3].

Then,

$$
\begin{aligned}
& i_{A_{H}, H} \circ b_{A} \circ \eta_{A} \\
= & \Omega_{A_{H} \otimes H} \circ\left(p_{A} \otimes H\right) \circ \rho_{A} \circ \eta_{A} \\
= & \left(\left(p_{A} \circ \mu_{A} \circ\left(i_{A} \otimes h\right)\right) \otimes H\right) \circ\left(p_{A} \otimes \delta_{H}\right) \circ \rho_{A} \circ \eta_{A} \\
= & \left(\left(p_{A} \circ \mu_{A} \circ\left(q_{A} \otimes h\right) \circ \rho_{A}\right) \otimes H\right) \circ \rho_{A} \circ \eta_{A} \\
= & \left(p_{A} \otimes H\right) \circ \rho_{A} \circ \eta_{A},
\end{aligned}
$$

where the first equality follows by the definition of $b_{A}$, the second one by (16), the third one by the properties of $\rho_{A}$, the fourth one by the equality (d2) of Lemma 3.9 of [3], i.e. $\mu_{A} \circ\left(q_{A} \otimes h\right) \circ \rho_{A}=i d_{A}$. On the other hand, using (16), the properties of $\eta_{A_{H}}$ and the condition of total integral for $h$ we have

$$
\begin{aligned}
& i_{A_{H}, H} \circ p_{A_{H}, H} \circ\left(\eta_{A_{H}} \otimes \eta_{H}\right) \\
= & \Omega_{A_{H} \otimes H} \circ\left(\eta_{A_{H}} \otimes \eta_{H}\right) \\
= & \left(p_{A} \otimes H\right) \circ \rho_{A} \circ h \circ \eta_{H}
\end{aligned}
$$




$$
=\left(p_{A} \otimes H\right) \circ \rho_{A} \circ \eta_{A} .
$$

Therefore, we get (d1), because $i_{A_{H}, H}$ is a monomorphism. Moreover,

$$
\begin{aligned}
& \left(\left(b_{A} \circ i_{A}\right) \otimes \varepsilon_{H}\right) \circ \Omega_{A_{H} \otimes H} \circ\left(\eta_{A_{H}} \otimes H\right) \\
= & b_{A} \circ i_{A} \circ p_{A} \circ h \\
= & b_{A} \circ q_{A} \circ h \\
= & b_{A} \circ\left(h * h^{-1}\right) \\
= & b_{A} \circ h \circ \bar{\Pi}_{H}^{L} \\
= & b_{A} \circ \omega_{A} \circ\left(\eta_{A_{H}} \otimes \bar{\Pi}_{H}^{L}\right) \\
= & p_{A_{H}, H} \circ \omega_{A}^{\prime} \circ \omega_{A} \circ\left(\eta_{A_{H}} \otimes \bar{\Pi}_{H}^{L}\right) \\
= & p_{A_{H}, H} \circ \Omega_{A_{H} \otimes H} \circ\left(\eta_{A_{H}} \otimes \bar{\Pi}_{H}^{L}\right) \\
= & p_{A_{H}, H} \circ\left(\eta_{A_{H}} \otimes \bar{\Pi}_{H}^{L}\right),
\end{aligned}
$$

and (d2) holds. Note that in the first equality we used (16), in the fourth one we applied that $h$ is total and in the fifth one we use that $\omega_{A} \circ\left(\eta_{A_{H}} \otimes H\right)=h$.

Therefore, $A_{H} \hookrightarrow A$ is an $H$-Galois extension with normal basis.

(ii) $\Rightarrow$ (i) Conversely, assume that $A_{H} \hookrightarrow A$ is an $H$-Galois extension with normal basis. Then, $A_{H} \hookrightarrow A$ is a weak $H$-Galois extension with normal basis and by Theorem 2.11 of [2] is a weak $H$-cleft extension with cleaving morphism $h=\omega_{A} \circ\left(\eta_{A} \otimes H\right)$ and $h^{-1}=m_{A} \circ \gamma_{A}^{-1} \circ p_{A \otimes H} \circ\left(\eta_{A} \otimes H\right)$. Therefore $h^{-1} * h=e_{A}$. Also $h * h^{-1}$ factorizes through the equalizer $i_{A}$.

By (13) and (d2) of Definition 2.7,

$$
\begin{aligned}
& h * h^{-1} \\
= & \left(i_{A} \otimes \varepsilon_{H}\right) \circ \Omega_{A_{H} \otimes H} \circ\left(\eta_{A_{H}} \otimes H\right) \\
= & b_{A}^{-1} \circ p_{A_{H}, H} \circ\left(\eta_{A_{H}} \otimes \bar{\Pi}_{H}^{L}\right) \\
= & \omega_{A} \circ\left(\eta_{A_{H}} \otimes \bar{\Pi}_{H}^{L}\right) \\
= & h \circ \bar{\Pi}_{H}^{L} .
\end{aligned}
$$

On the other hand, using that $b_{A}$ and $\Omega_{A_{H} \otimes H}$ are morphisms of right $H$ comodules and (d1) of Definition 2.7 we have:

$$
\begin{aligned}
& \left(A \otimes\left(\varepsilon_{H} \circ \mu_{H}\right)\right) \circ\left(\left(\rho_{A} \circ \eta_{A}\right) \otimes H\right) \\
= & \left(\left(b_{A}^{-1} \circ b_{A}\right) \otimes\left(\varepsilon_{H} \circ \mu_{H}\right)\right) \circ\left(\left(\rho_{A} \circ \eta_{A}\right) \otimes H\right) \\
= & \left(b_{A}^{-1} \otimes\left(\varepsilon_{H} \circ \mu_{H}\right)\right) \circ\left(\left(\rho_{A_{H} \times H} \circ b_{A} \circ \eta_{A}\right) \otimes H\right) \\
= & \left(\left(b_{A}^{-1} \circ p_{A_{H}, H}\right) \otimes\left(\varepsilon_{H} \circ \mu_{H}\right)\right) \circ\left(\left(\left(A_{H} \otimes \delta_{H}\right) \circ \Omega_{A_{H} \otimes H} \circ\left(\eta_{A_{H}} \otimes \eta_{H}\right)\right) \otimes H\right) \\
= & b_{A}^{-1} \circ p_{A_{H}, H} \circ\left(\eta_{A_{H}} \otimes \bar{\Pi}_{H}^{L}\right) \\
= & \omega_{A} \circ\left(\eta_{A_{H}} \otimes \bar{\Pi}_{H}^{L}\right) \\
= & h \circ \bar{\Pi}_{H}^{L},
\end{aligned}
$$

where the third equality uses the definitions of $\Omega_{A_{H} \otimes H}$ and $\rho_{A_{H} \times H}$. 
Therefore, $h * h^{-1}=\left(A \otimes\left(\varepsilon_{H} \circ \mu_{H}\right)\right) \circ\left(\left(\rho_{A} \circ \eta_{A}\right) \otimes H\right)$ and $h * h^{-1}=h \circ \bar{\Pi}_{H}^{L}$. As a consequence,

$$
h \circ \eta_{H}=h \circ \bar{\Pi}_{H}^{L} \circ \eta_{H}=\left(A \otimes\left(\varepsilon_{H} \circ \mu_{H}\right)\right) \circ\left(\left(\rho_{A} \circ \eta_{A}\right) \otimes \eta_{H}\right)=\eta_{A},
$$

and $h$ is a total integral.

To finish the proof it only remains to show that $h^{-1} * h * h^{-1}=h^{-1}$. First we proceed by showing the identity

$$
m_{A}=\mu_{A} \circ\left(m_{A} \otimes\left(h \circ \bar{\Pi}_{H}^{L}\right)\right) \circ \rho_{A \otimes_{A_{H}} A} .
$$

Indeed, composing with the coequalizer $q_{A, A}$ we have that

$$
\begin{aligned}
& \mu_{A} \circ\left(m_{A} \otimes\left(h \circ \bar{\Pi}_{H}^{L}\right)\right) \circ \rho_{A \otimes A_{H} A} \circ q_{A, A} \\
= & \mu_{A} \circ\left(\left(m_{A} \circ q_{A, A}\right) \otimes\left(h \circ \bar{\Pi}_{H}^{L}\right)\right) \circ\left(A \otimes \rho_{A}\right) \\
= & \mu_{A} \circ\left(\mu_{A} \otimes A\right) \circ\left(A \otimes\left(\left(i_{A} \otimes \varepsilon_{H} \otimes\left(h \circ \bar{\Pi}_{H}^{L}\right)\right) \circ\left(\omega_{A}^{\prime} \otimes H\right) \circ \rho_{A}\right)\right) \\
= & \mu_{A} \circ\left(\mu_{A} \otimes A\right) \circ\left(A \otimes\left(\left(i_{A} \otimes\left(\varepsilon_{H} \otimes\left(h \circ \bar{\Pi}_{H}^{L}\right) \circ \delta_{H}\right)\right) \circ \omega_{A}^{\prime}\right)\right) \\
= & \mu_{A} \circ\left(\mu_{A} \otimes A\right) \circ\left(A \otimes\left(\left(i_{A} \otimes\left(h \circ \bar{\Pi}_{H}^{L}\right)\right) \circ \omega_{A}^{\prime}\right)\right) \\
= & \mu_{A} \circ\left(\mu_{A} \otimes A\right) \circ\left(A \otimes\left(\left(i_{A} \otimes\left(\left(i_{A} \otimes \varepsilon_{H}\right) \circ \Omega_{A_{H} \otimes H} \circ\left(\eta_{A_{H}} \otimes H\right)\right)\right) \circ \omega_{A}^{\prime}\right)\right) \\
= & \mu_{A} \circ\left(A \otimes i_{A}\right) \circ\left(A \otimes\left(\left(\mu_{A_{H}} \otimes \varepsilon_{H}\right) \circ\left(A_{H} \otimes\left(\Omega_{A_{H} \otimes H} \circ\left(\eta_{A_{H}} \otimes H\right)\right)\right) \circ \omega_{A}^{\prime}\right)\right) \\
= & \mu_{A} \circ\left(A \otimes\left(\left(i_{A} \otimes \varepsilon_{H}\right) \circ \Omega_{A_{H} \otimes H} \circ \omega_{A}^{\prime}\right)\right) \\
= & \mu_{A} \circ\left(A \otimes\left(\left(i_{A} \otimes \varepsilon_{H}\right) \circ \omega_{A}^{\prime}\right)\right) \\
= & m_{A} \circ q_{A, A},
\end{aligned}
$$

and then (17) holds. In the last equalities, the first one follows by the definition of $\rho_{A \otimes_{A_{H}} A}$, the second one by (12) and in the third one we used that $\omega_{A}^{\prime}$ is a morphism of right $H$-comodules. The fourth equality follows by the properties of the counit and the fifth one by the identity

$$
h \circ \bar{\Pi}_{H}^{L}=\left(i_{A} \otimes \varepsilon_{H}\right) \circ \Omega_{A_{H} \otimes H} \circ\left(\eta_{A_{H}} \otimes H\right),
$$

obtained in the proof that $h * h^{-1}=h \circ \bar{\Pi}_{H}^{L}$. The sixth one relies on the definition of $\mu_{A_{H}}$, in the seventh one we applied that $\Omega_{A_{H} \otimes H}$ is a morphism of left $A_{H}$-modules and in the eighth one we used that $\Omega_{A_{H} \otimes H}=\omega_{A}^{\prime} \circ \omega_{A}$ and $\omega_{A} \circ \omega_{A}^{\prime}=i d_{A}$. Finally, the last one follows by (12).

Therefore,

$$
\begin{aligned}
& h^{-1} * h * h^{-1} \\
= & \mu_{A} \circ\left(h^{-1} \otimes\left(h \circ \bar{\Pi}_{H}^{L}\right)\right) \circ \delta_{H} \\
= & \mu_{A} \circ\left(\left(m_{A} \circ \gamma_{A}^{-1} \circ p_{A \otimes H}\right) \otimes\left(h \circ \bar{\Pi}_{H}^{L}\right)\right) \circ\left(A \otimes \delta_{H}\right) \circ \Delta_{A \otimes H} \circ\left(\eta_{A} \otimes H\right) \\
= & \mu_{A} \circ\left(\left(m_{A} \circ \gamma_{A}^{-1}\right) \otimes\left(h \circ \bar{\Pi}_{H}^{L}\right)\right) \circ \rho_{A \square H} \circ p_{A \otimes H} \circ\left(\eta_{A} \otimes H\right) \\
= & \mu_{A} \circ\left(m_{A} \otimes\left(h \circ \bar{\Pi}_{H}^{L}\right)\right) \circ \rho_{A \otimes A_{H}} \circ \gamma_{A}^{-1} \circ p_{A \otimes H} \circ\left(\eta_{A} \otimes H\right) \\
= & m_{A} \circ \gamma_{A}^{-1} \circ p_{A \otimes H} \circ\left(\eta_{A} \otimes H\right) \\
= & h^{-1},
\end{aligned}
$$


where the first equality follows using that $h * h^{-1}=h \circ \bar{\Pi}_{H}^{L}$, the second one because $\Delta_{A \otimes H}$ is a morphism of right $H$-comodules, the third one by the definition of $\rho_{A \square H}$ and in the fourth one we applied that $\gamma_{A}^{-1}$ is a morphism of right $H$-comodules. Finally, the fifth equality follows by (17) and the last one by the definition of $h^{-1}$.

As a consequence, by Propositions 2.2 and 2.3 of [5], we have the following corollary.

Corollary 3.3. Let $H$ be a cocommutative weak Hopf algebra and let $\left(A, \rho_{A}\right)$ be a right $H$-comodule algebra such that $A \otimes-$ preserves coequalizers. The following are equivalent.

(i) $A_{H} \hookrightarrow A$ is an $H$-cleft extension.

(ii) $A_{H} \hookrightarrow A$ is an $H$-Galois extension with normal basis.

To finish this paper, we will give a cohomological interpretation of Cleft extensions. For clarity, we briefly describe the construction of the cohomology groups in the weak setting. The interested reader can find the details in [4]. Assume that $H$ is a cocommutative weak Hopf algebra and let $\left(A, \varphi_{A}\right)$ be a commutative weak left $H$-module algebra. Let $H^{0}$ be the unit object of $\mathcal{C}$ and for $n \geq 1$ denote by $H^{n}$ the $n$-fold tensor power $H \otimes \cdots \otimes H$. If $n \geq 2, m_{H}^{n}$ denotes the morphism $m_{H}^{n}: H^{n} \rightarrow H$ defined by $m_{H}^{2}=\mu_{H}$ and by $m_{H}^{3}=m_{H}^{2} \circ\left(H \otimes \mu_{H}\right), \cdots, m_{H}^{n}=m_{H}^{n-1} \circ\left(H^{n-2} \otimes \mu_{H}\right)$ for $k>2$. Analogously, with $\delta_{H^{n}}$ we denote the coproduct defined for the coalgebra $H^{n}$. Finally, $\varphi_{A}^{n}$ will be the morphism $\varphi_{A}^{n}: H^{n} \otimes A \rightarrow A$ defined as $\varphi_{A}^{1}=\varphi_{A}$ and $\varphi_{A}^{n}=\varphi_{A} \circ\left(H \otimes \varphi_{A}^{n-1}\right)$. For brevity, we denote the morphisms $\varphi_{A} \circ\left(m_{H}^{n} \otimes \eta_{A}\right)$ and $\varphi_{A} \circ\left(H \otimes \eta_{A}\right)$ by $u_{n}$ and $u_{1}$, respectively.

For $n \geq 1$, let $\operatorname{Reg}_{\varphi_{A}}\left(H^{n}, A\right)$ be the set of morphisms $\sigma: H^{n} \rightarrow A$ such that there exists a morphism $\sigma^{-1}: H^{n} \rightarrow A$ (the convolution inverse of $\sigma$ ) satisfying the following equalities:

(c1) $\sigma \wedge \sigma^{-1}=\sigma^{-1} \wedge \sigma=u_{n}$.

(c2) $\sigma \wedge \sigma^{-1} \wedge \sigma=\sigma$.

(c3) $\sigma^{-1} \wedge \sigma \wedge \sigma^{-1}=\sigma^{-1}$,

and $\operatorname{Reg}_{\varphi_{A}}\left(H_{L}, A\right)$ will be the set of morphisms $g: H_{L} \rightarrow A$ such that there exists a morphism $g^{-1}: H_{L} \rightarrow A$ satisfying

$$
g \wedge g^{-1}=g^{-1} \wedge g=u_{0}, \quad g \wedge g^{-1} \wedge g=g, \quad g^{-1} \wedge g \wedge g^{-1}=g^{-1}
$$

where $u_{0}=u_{1} \circ i_{L}$.

The sets $\operatorname{Reg}_{\varphi_{A}}\left(H_{L}, A\right), \operatorname{Reg}_{\varphi_{A}}\left(H^{n}, A\right)$ are abelian groups with neutral elements $u_{0}$ and $u_{n}$ respectively. Moreover, we can define a cosimplicial complex of abelian 
groups with coface operators defined by

$$
\begin{gathered}
\partial_{0, i}: \operatorname{Reg}_{\varphi_{A}}\left(H_{L}, A\right) \rightarrow \operatorname{Reg}_{\varphi_{A}}(H, A), \quad i \in\{0,1\} \\
\partial_{0,0}(g)=\varphi_{A} \circ\left(H \otimes\left(g \circ p_{L} \circ \Pi_{H}^{R}\right)\right) \circ \delta_{H}, \quad \partial_{0,1}(g)=g \circ p_{L}, \\
\partial_{k-1, i}: \operatorname{Reg}_{\varphi_{A}}\left(H^{k-1}, A\right) \rightarrow \operatorname{Reg}_{\varphi_{A}}\left(H^{k}, A\right), \quad k \geq 2, \quad i \in\{0,1, \cdots, k\} \\
\partial_{k-1, i}(\sigma)=\left\{\begin{array}{l}
\varphi_{A} \circ(H \otimes \sigma), \quad i=0 \\
\sigma \circ\left(H^{i-1} \otimes \mu_{H} \otimes H^{k-i-1}\right), \quad i \in\{1, \cdots, k-1\} \\
\sigma \circ\left(H^{k-2} \otimes\left(\mu_{H} \circ\left(H \otimes \Pi_{H}^{L}\right)\right)\right), i=k,
\end{array}\right.
\end{gathered}
$$

and codegeneracy operators defined by $s_{1,0}: \operatorname{Reg}_{\varphi_{A}}(H, A) \rightarrow \operatorname{Reg}_{\varphi_{A}}\left(H_{L}, A\right)$,

$$
s_{1,0}(h)=h \circ i_{L}
$$

and $s_{k+1, i}: \operatorname{Reg}_{\varphi_{A}}\left(H^{k+1}, A\right) \rightarrow \operatorname{Reg}_{\varphi_{A}}\left(H^{k}, A\right), \quad k \geq 1, \quad i \in\{0,1, \cdots, k\}$

$$
s_{k+1, i}(\sigma)=\sigma \circ\left(H^{i} \otimes \eta_{H} \otimes H^{k-i}\right) .
$$

Let $D_{\varphi_{A}}^{k}=\partial_{k, 0} \wedge \partial_{k, 1}^{-1} \wedge \cdots \wedge \partial_{k, k+1}^{(-1)^{k+1}}$ be the coboundary morphisms of the cochain complex

$$
\begin{aligned}
\operatorname{Reg}_{\varphi_{A}}\left(H_{L}, A\right) & \stackrel{D_{\varphi_{A}}^{0}}{\longrightarrow} \operatorname{Reg}_{\varphi_{A}}(H, A) \stackrel{D_{\varphi_{A}}^{1}}{\longrightarrow} \operatorname{Reg}_{\varphi_{A}}\left(H^{2}, A\right) \stackrel{D_{\varphi_{A}}^{2}}{\longrightarrow} \cdots \\
\ldots & \stackrel{D_{\varphi_{A}}^{k-1}}{\longrightarrow} \operatorname{Reg}_{\varphi_{A}}\left(H^{k}, A\right) \stackrel{D_{\varphi_{A}}^{k}}{\longrightarrow} \operatorname{Reg}_{\varphi_{A}}\left(H^{k+1}, A\right) \stackrel{D_{\varphi_{A}}^{k+1}}{\longrightarrow} \cdots
\end{aligned}
$$

associated to the cosimplicial complex $\operatorname{Reg}_{\varphi_{A}}\left(H^{\bullet}, A\right)$.

Then, $\left(\operatorname{Reg}_{\varphi_{A}}\left(H^{\bullet}, A\right), D_{\varphi_{A}}^{\bullet}\right)$ gives the Sweedler cohomology of $H$ in $\left(A, \varphi_{A}\right)$. Therefore, the kth group will be defined by

$$
H_{\varphi_{A}}^{k}(H, A)=\frac{\operatorname{Ker}\left(D_{\varphi_{A}}^{k}\right)}{\operatorname{Im}\left(D_{\varphi_{A}}^{k-1}\right)}
$$

for $k \geq 1$ and $\operatorname{Ker}\left(D_{\varphi_{A}}^{0}\right)$ for $k=0$.

Remark 3.4. Let $H$ be a weak Hopf algebra. Two $H$-cleft extensions $A_{H} \hookrightarrow A$ and $B_{H} \hookrightarrow B$ are equivalent if $A_{H}=B_{H}$ and there exists a morphism of right $H$-comodule algebras $T$ such that $T \circ i_{A}=i_{B}$. Under these conditions $T$ is an isomorphism (see Definition 2.13 of [5]). If $H$ is cocommutative and $h$ is a convolution invertible total integral for an $H$-cleft extension $A_{H} \hookrightarrow A$, by Proposition 2.18 of [5], we know that $\left(A_{H}, \varphi_{A_{H}}=p_{A} \circ \mu_{A} \circ\left(h \otimes i_{A}\right)\right)$ is a left $H$-module algebra and, if $\sigma_{A_{H}}=p_{A} \circ \mu_{A} \circ(h \otimes h)$, the pair $\left(\varphi_{A_{H}}, \sigma_{A_{H}}\right)$ is a crossed system for $H$ over $A_{H}$ (see Definition 3.2 and Theorem 3.2 of [5]). If we denote by $\operatorname{Cleft}\left(A_{H}, \varphi_{A_{H}}\right)$ the set of equivalence classes of $H$-cleft extensions with a fixed structure $\varphi_{A_{H}}$, by Corollary 3.3 of [5], we obtain that there exists a bijective correspondence between 
$\operatorname{Cleft}\left(A_{H}, \varphi_{A_{H}}\right)$ and the set of equivalence classes of crossed systems for $H$ over $A_{H}$ with a common action $\varphi_{A_{H}}$. Therefore, if $A$ is commutative, there exists a bijective correspondence between the second cohomology group $H_{\varphi_{A_{H}}}^{2}\left(H, A_{H}\right)$, defined in [4], and Cleft $\left(A_{H}, \varphi_{A_{H}}\right)$ (see Theorem 4.1 of [5]). As a consequence, by Corollary 3.3, there exists a bijection between $H_{\varphi_{A_{H}}}^{2}\left(H, A_{H}\right)$ and the set of equivalence classes of $H$-Galois extensions with normal basis $A_{H} \hookrightarrow A$ such that for their associated convolution invertible total integrals $h$ we have $\varphi_{A_{H}}=p_{A} \circ \mu_{A} \circ\left(h \otimes i_{A}\right)$ (the equivalence relation between $H$-Galois extensions with normal basis is the one induced by the equivalence relation between $H$-cleft extensions).

For example, if $H$ is cocommutative, $H_{L}$ is commutative and $\left(H_{L}, \varphi_{H_{L}}=p_{L} \circ\right.$ $\left.\mu_{H} \circ\left(H \otimes i_{L}\right)\right)$ is a left $H$-module algebra. Therefore, there exists a bijection between

$H_{\varphi_{H_{L}}}^{2}\left(H, H_{L}\right)$ and the set of equivalence classes of $H$-Galois extensions with normal basis $H_{L} \hookrightarrow A$ such that $\varphi_{H_{L}}=p_{A} \circ \mu_{A} \circ\left(h \otimes i_{A}\right)$. In the Hopf algebra setting, if $H$ is a finite Hopf algebra, the last bijection is the isomorphism between the second cohomology group $H^{2}(H, K)$, introduced by Sweedler [14], and the group of isomorphism classes of Galois $H$-objects with normal basis.

\section{References}

[1] J. N. Alonso Álvarez, J. M. Fernández Vilaboa, R. González Rodríguez and A. B. Rodríguez Raposo, Weak C-cleft extensions, weak entwining structures and weak Hopf algebras, J. Algebra, 284(2) (2005), 679-704.

[2] J. N. Alonso Álvarez, J. M. Fernández Vilaboa, R. González Rodríguez and A. B. Rodríguez Raposo, Weak C-cleft extensions and weak Galois extensions, J. Algebra, 299(1) (2006), 276-293.

[3] J. N. Alonso Álvarez, J. M. Fernández Vilaboa, R. González Rodríguez and A. B. Rodríguez Raposo, Crossed products in weak contexts, Appl. Categ. Structures, 18(3) (2010), 231-258.

[4] J. N. Alonso Álvarez, J. M. Fernández Vilaboa and R. González Rodríguez, Cohomology of algebras over weak Hopf algebras, Homology Homotopy Appl., 16(1) (2014), 341-369.

[5] J. N. Alonso Álvarez, J. M. Fernández Vilaboa and R. González Rodríguez, Crossed products over weak Hopf algebras related to cleft extensions and cohomology, Chin. Ann. Math. Ser. B, 35(2) (2014), 161-190.

[6] J. N. Alonso Álvarez, J. M. Fernández Vilaboa and R. González Rodríguez, Weak Hopf quasigroups, to appear in Asian J. Math., arXiv:1410.2180 (2014).

[7] T. Brzeziński, On modules associated to coalgebra Galois extensions, J. Algebra, 215(1) (1999), 290-317. 
[8] S. Caenepeel and E. de Groot, Modules over weak entwining structures, Contemp. Math., 267 (2000), 31-54.

[9] Y. Doi, Equivalent crossed products for a Hopf algebra, Comm. Algebra, 17(12) (1989), 3053-3085.

[10] Y. Doi and M. Takeuchi, Cleft comodule algebras for a bialgebra, Comm. Algebra, 14(5) (1986), 801-817.

[11] J. M. Fernández Vilaboa and E. Villanueva Novoa, A characterization of the cleft comodule triples, Comm. Algebra, 16(3) (1988), 613-622.

[12] C. Kassel, Quantum Groups, Graduate Texts in Mathematics, 155, SpringerVerlag, New York, 1995.

[13] H. F. Kreimer and M. Takeuchi, Hopf algebras and Galois extensions of an algebra, Indiana Univ. Math. J., 30(5) (1981), 675-692.

[14] M. E. Sweedler, Cohomology of algebras over Hopf algebras, Trans. Amer. Math. Soc., 133 (1968), 205-239.

J. N. Alonso Álvarez (Corresponding Author)

Departamento de Matemáticas

Universidad de Vigo

Campus Universitario Lagoas-Marcosende

E-36280 Vigo, Spain

e-mail: jnalonso@uvigo.es

\section{J. M. Fernández Vilaboa}

Departamento de Álxebra

Universidad de Santiago de Compostela

Campus Sur

E-15771 Santiago de Compostela, Spain

e-mail: josemanuel.fernandez@usc.es

\section{R. González Rodríguez}

Departamento de Matemática Aplicada II Universidad de Vigo

Campus Universitario Lagoas-Marcosende

E-36310 Vigo, Spain

e-mail: rgon@dma.uvigo.es 